\title{
Anti-Biofilm Strategies: How to Eradicate Candida Biofilms?
}

\author{
A. Bink, K. Pellens, B. P.A. Cammue, and K. Thevissen ${ }^{*}$ \\ Centre of Microbial and Plant Genetics, Katholieke Universiteit Leuven, Kasteelpark Arenberg 20, 3001 Heverlee- \\ Leuven, Belgium
}

\begin{abstract}
In nature, microorganisms prefer to reside in structured microbial communities, termed biofilms, rather than as free-floating planktonic cells. Advantageous for the microorganisms, but disadvantageous for human health, is the increased resistance/tolerance of the biofilm cells to antimicrobial treatment. In clinically relevant biofilms, Candida albicans is one of the most frequently isolated microorganisms in biofilms. This review primarily elaborates on the activity of the currently used antimycotics against Candida biofilms, the potential of antifungal lock therapy and sheds more light on new promising compounds resulting from the gradual shift of anti-biofilm research activities to natural products, plants and their extracts.
\end{abstract}

Keywords: Candida, antifungal therapy, lock therapy, novel anti-biofilm agents.

\section{INTRODUCTION}

One of the reasons for the growing frequency of hospital acquired Candida bloodstream infections is the increasing use of immunosuppresive therapy in cancer and transplant patients, which leads to breakdown of the barrier between the gut and bloodstream [1]. Candida cells, like many other microbial organisms, are able to adhere to and colonize surfaces of medical devices, like central venous catheters, voice prostheses, intrauterine devices and prosthetic joints, among others, resulting in the development of a biofilm. Evidence for the occurrence of Candida biofilms on surfaces comes from various in vivo studies, in which the devices are examined upon removal out of the patients, or from animal model systems. Techniques such as scanning electron microscopy, confocal laser scanning microscopy and echocardiography (to demonstrate the occurrence of biofilms on heart valves) can be used for biofilm visualization [2-4].

Infections due to the presence of fungal biofilms are a major clinical concern as these structured microbial communities, embedded in an extracellular matrix, are characterized by increased resistance to antifungal therapy [5]. In many cases, the implant has to be removed in order to cure the infection. A combined action of different mechanisms is believed to contribute to increased resistance: (i) amplified expression of efflux pumps, (ii) a changing sterol composition in the membrane, (iii) limited diffusion of molecules through the extracellular matrix and (iv) the presence of persisters in the biofilm, which are able to tolerate high concentrations of antimycotics [6]. Interestingly, these persisters are not mutants but rather phenotypical variants of wild type cells [7]. Until now, the molecular basis of persistence in C. albicans biofims is not known [8].

This review focuses on the treatment options for biofilm associated Candida infections, as well as on novel antibiofilm compounds or therapy strategies.

*Address correspondence to this author at the Centre of Microbial and Plant Genetics, Katholieke Universiteit Leuven, Kasteelpark Arenberg 20, 3001 Heverlee-Leuven, Belgium; Tel: +32 163296 88; Fax: +32 163219 66; E-mail: Karin.Thevissen@biw.kuleuven.be

\section{CURRENT THERAPEUTIC OPTIONS}

The current treatment options for fungal biofilm-related infections are very scarce due to the intrinsic increased tolerance of biofilms to antimycotics. In the mid 1990s, $C$. albicans biofilms were found to be resistant to the majority of the antifungal agents [9]. Patients with fungal biofilminfected devices are rarely cured with mono-antifungal therapy and affected devices generally need to be removed [10-15]. Percutaneous vascular catheters may be removed quickly. However, the removal of infected heart valves, joint prostheses, central nervous system shunts and other implanted medical devices is problematic because these implants generally have a life-supportive function. Hence, succesful treatment, thereby retaining the implanted devices, are urgently needed in clinical practice.

\section{Conventional Antimycotics}

It has been more than a decade since the first reports were published regarding a near-total in vitro resistance of Candida biofilms to antifungal agents $[9,16,17]$. To this end, a variety of in vitro biofilm model systems have been developed including in vitro biofilm formation on the surface of small catheter material (polyvinyl chloride) discs, on denture acrylic strips or on silicone elastomer discs [17-19]. The reader is directed to the review by Coenye et al. in this special issue of Open Mycology for more information in this regard.

Azoles

An early report by Hawser and Douglas investigated the susceptibility of Candida biofilms grown statically on small polyvinyl chloride discs, to a number of clinically relevant antifungal agents. They demonstrated that fluconazole (a triazole) was most potent, while amphotericin B showed only moderate activity against biofilm cells, similar to the effect of itraconazole (a triazole), ketoconazole (an imidazole) and flucytosine. Although fluconazole proved to be the most effective agent against Candida biofilms, the concentration corresponding to $50 \%$ inhibition was 28 to 38 
times higher than the relevant MICs [9]. However, in 2004, in vitro mature $C$. albicans biofilms were found to be highly resistant to fluconazole and miconazole (an imidazole). In this study, biofilms were grown on denture acrylic discs in a constant depth film fermentor and maintained with artificial saliva to simulate biofilm formation in the oral cavity. Early phase $C$. albicans biofilms (2-6h) proved to be susceptible to fluconazole $(256 \mathrm{mg} / \mathrm{l})$ and miconazole $(256 \mathrm{mg} / \mathrm{l})$ in this model system, with a reduction of $>83 \%$ and $>99 \%$, respectively, in viability after $24 \mathrm{~h}$ biofilm exposure [20]. Very recently, the group of Coenye also demonstrated fungicidal activity of miconazole against mature Candida species biofilms [21]. In the latter study, Candida biofilms were grown statically on silicone discs $(24 \mathrm{~h})$ whereafter they were exposed to $5 \mathrm{mM}$ miconazole (corresponding to 2081 $\mathrm{mg} / \mathrm{l}$ ) during $24 \mathrm{~h}$. A substantial reduction, ranging from $89.3 \%$ to $99.1 \%$, in the number of colony forming units recovered from the discs, was observed. The concentration miconazole applied in these in vitro experiments was higher than the commonly used therapeutic concentration, but is achievable during, for instance, antifungal lock therapy. Treatment of mature Candida biofilms with $5 \mathrm{mM}$ fluconazole only resulted in a fungistatic effect [21]. Hence, depending on the in vitro system used, data on biofilm susceptibility differ substantially.

The azole antifungals were discovered about 30 years ago and refinements to the azole class led to agents with a triazole at their core. As the first-generation triazoles fluconazole and itraconazole have limitations related to their spectrum of antifungal activity and their tolerability, there have been efforts to develop new triazoles that address these limitations and have led to the regulatory approvals of voriconazole (approved by the FDA in 2002) and posaconazole (approved by the FDA in 2006). However, these newer azoles are not active against $C$. albicans or $C$. parapsilosis biofilms formed on silicone elastomer discs [22].

\section{Polyenes}

In line with the above data, Kuhn and coworkers confirmed the resistance of mature C. albicans, and also of $C$. parapsilosis biofilms, to conventional agents such as fluconazole and amphotericin B. They however showed that the newer lipid formulations of amphotericin B (liposomal amphotericin B and amphotericin B lipid complex) exhibited inhibitory activities against mature $C$. albicans biofilms, grown on silicone elastomer discs. The antifungal concentration resulting in $50 \%$ reduction in metabolic activity of the biofilm [23] was equivalent to the respective MIC50-value for planktonic cells [19]. Recently it was shown that liposomal amphotericin B, at its MIC $(0.5 \mu \mathrm{g} / \mathrm{ml})$ is also able to eradicate $C$. albicans biofilms in a novel continuous catheter flow model [24]. Using a newly developed in vivo rabit biofilm model, Shuford and coworkers assessed the in vivo activity of amphotericin B and caspofungin (see echinocandin section below) against Candida biofilm growth on central venous catheters. They demonstrated that systemic therapy with either caspofungin or amphotericin B deoxycholate and combined with intraluminal lock therapy (see further), significantly reduced colony counts of C. albicans associated with catheters and even resulting in sterile catheters in many cases [25]. Another study demonstrated that amphotericin B lipid complex was able to sterilise catheters on which $C$. albicans biofilms were formed, using a rabbit model of catheter-associated candidal biofilm [26].

Like amphotericin $\mathrm{B}$, the polyene nystatin is one of the antifungal drugs most commenly used to treat patients with oral fungal infections topically. Susceptibility testing of nystatin, revealed resistance in all Candida isolates examined by Khun and coworkers, when grown as biofilms [19]. However, incorporation of nystatin into a thin-film polymer on deture material reduced Candida biofilm formation with $70-80 \%$ whereas this was $50-60 \%$ in case of coating with amphotericin B. This novel thin-film coating with various antifungals effectively inhibits $C$. albicans biofilm formation and should be evaluated as a potential preventive therapy for denture stomatitis [27]. Very recently, De Prijck and coworkers investigated the effect of nystatin released from modified polydimethyl siloxane disk as a model for incorporating antifungals in medical devices against biofilm formation by Candida spp. Nystatin exhibited a concentration-dependent inhibitory effect on Candida biofilm formation in a microtiter plate but not in a Modified Robbins Device [28]. The small fraction of free released nystatin killed $C$. albicans biofilm cells in the limited volume of a microtiter plate well but not in the flow system [29].

\section{Echinocandins}

Kuhn and colleagues further demonstrated that caspofungin and micafungin, both members of the more recent class of echinocandin antifungals, are active against biofilmassociated C. albicans [19]. A few months after their report, Bachmann and colleagues published similar results regarding the activity of caspofungin against $C$. albicans biofilms. In this case, biofilms were formed in wells of microtiter plates [30]. More than $97 \%$ reduction of the metabolic activity of sessile cells was observed after treatment with caspofungin concentrations well within its therapeutic range $(0.125$ $\mu \mathrm{g} / \mathrm{ml})[31,32]$. The antifungal activity of caspofungin on Candida biofilms was also studied in relation to the biofilm maturation age and the yeast susceptibility to fluconazole. It appeared that the activity of caspofungin applied at MIC dose against Candida biofilms formed on sections of silicone catheters was species-dependent (i.e. $C$. albicans or $C$. parapsilosis). Moreover, caspofungin activity at its MIC depends on maturation age of the C. parapsilosis biofilms. However, the inhibition of biofilm metabolic activity caused by $2 \mathrm{mg} / \mathrm{l}$ caspofungin (which is within the therapeutic range) was not species-specific, independent on the state of biofilm maturation and not affected by the yeast's resistance to fluconazole [33]. In line with the above activity of micafungin against $C$. albicans biofilm [19], another research group confirmed this activity against $C$. albicans biofilms, in this case grown on central venous catheter sections, and extended the observations to biofilms formed by C. glabrata and C. parapsilosis [34]. In contrast, Choi and coworkers showed that within the therapeutic concentration range of micafungin and caspofungin, both drugs are active against $C$. albicans and $C$. glabrata biofilms, but not against biofilms formed by bloodstream isolates of $C$. tropicalis or C. parapsilosis [35]. In conclusion, these interstudy variations highlight the differences in susceptibilities of Candida biofilms, which can be due to differences in the 
biofilm-forming abilities of the Candida isolates tested or to the diversity of the biofilm models used.

In 2006, the FDA approved the new systemic echinocandin anidulafungin and two years later the first publication on its efficacy against Candida biofilms appeared [22]. Anidulafungin, like caspofungin and micafungin, showed enhanced potency against $C$. albicans biofilms formed on silicone elastomer discs at clinically relevant concentrations. The efficacy of caspofungin against $C$. albicans biofilms in vivo was further demonstrated in a model for central venous catheter-associated candidiasis in mice. Treatment with 0.25 $\mu \mathrm{g} / \mathrm{ml}$ caspofungin (instilled in the catheter and allowed to dwell for 24h) significantly diminished the biofilm fungal load in the catheters and the dissemination to the kidneys compared with untreated controls [36]. Recently, the efficacy of anidulafungin against in vivo mature $C$. albicans biofilms was demonstrated using a newly developed rat model of catheter-associated candidiasis. The model was based on the avascular implantation of small polyurethane catheters challenged with Candida cells prior to their implantation in immunosuppressed rats. The model allowed the study of up to 10 biofilms at once, and the recovery of mature biofilms from 2 days after implantation. The adhering inoculum was adjusted to the standard threshold of positive diagnosis of fungal infection in materials recovered from patients. Wild type biofilms were mainly composed of hyphal cells, and they were unevenly distributed across the catheter length as observed in infected materials in clinical cases [10]. This model can be used to characterize the ability of antimicrobial agents to eliminate biofilms, and to evaluate the prophylactic effect of antifungal drugs and biomaterial coatings [37,38]. In the latter case, anidulafungin was daily administered intraperitoneally at $10 \mathrm{mg} / \mathrm{kg}$ for 7 days. More than $70 \%$ of explanted catheters from anidulafungin-treated animals contained fewer than $2 \log _{10}$ colony forming units of $C$. albicans cells which was below the diagnostic threshold for catheter related infections [10]. In addition, $17 \%$ of the catheters from anidulafungin-treated animals were sterile [38]. In conclusion, Candida biofilms are intrinsically resistant to most antifungal drugs such as azoles (with the exception of miconazole). However, the lipid formulations of amphotericin B and the echinocandins do have activity against Candida biofilms.

\section{Antifungal Combination Therapy}

Efforts to successful treatment of Candida biofilmassociated infections are urgently needed in clinical practice. Combining antifungal drugs was recommended as a means to enhance efficacy in a variety of invasive infections including cryptococcosis, candidiasis, and aspergillosis. There are several foreseeable advantages to combination antifungal therapy, (i) a widened spectrum and potency of drug activity, (ii) more rapid antifungal effect, (iii) synergy, (iv) lowered dosing of toxic drug and (v) reduced risk of antifungal resistance. Utilizing agents with different mechanisms of action is a hallmark in current medical therapies in numerous medical disciplines, but of course one has to be cautious of some combinations as they may be antagonistic or clinically indifferent with additive side effects. Prior to the availability of echinocandins and azoles, the polyene antifungal agent amphotericin B was the major antifungal agent used in the management of invasive Candida infections. The high incidence of nephrotoxicity of amphotericin B led to the evaluation of combination therapies, to reduce the concentration and the toxicity required for treatment [39]. In 2002, Khun and coworkers demonstrated for the first time the unique activity of lipid formulations of amphotericin B and echinocandins against Candida biofilms [19]. Because in vivo and clinical studies are hard to perform, most of the reports below deal with in vitro studies of biofilm susceptibility following antifungal combination treatment. Synergism or antagonism of antifungal combinations is most commonly determined by the checkerboard method [40,41]. Bachmann and coworkers described in 2003 the indifferent interaction of either paired combination of the antifungals amphotericin $\mathrm{B}$, caspofungin and fluconazole against biofilms of a single C. albicans isolate in vitro. They demonstrated further an antagonistic effect by time-kill experiments of high fluconazole doses and caspofungin [42]. The combination caspofungin and voriconazole did not provide either an enhanced activity compared with caspofungin alone against biofilms of 30 clinical invasive strains of $C$. albicans associated with human infection [43]. However, very recently, Tobudic and coworkers investigated the in vitro activity and synergism of the combination of amphotericin B and caspofungin on one hand, and amphotericin B and posaconazole on the other hand against $C$. albicans biofilm cells. They reported that the combination of amphotericin B/pozaconazole yielded synergism, whereas amphotericin $\mathrm{B} /$ caspofungin yielded indifferent interactions against biofilms of various $C$. albicans isolates [44]. The indifference of amphotericin $\mathrm{B} /$ caspofungin is in line with the previous data of Bachmann and coworkers [42]. In addition, Pai and coworkers performed studies of various antifungal combinations against simulated Candida endocardial vegetations $[45,46]$. Current medical management includes combination use of amphotericin B and flucytosine followed by prolonged suppression with fluconazole $[47,48]$. However, multidrug resistance to longterm suppressive therapy can develop [49]. The low incidence and high morbidity and mortality associated with this disease demands alternative research strategies to improve current care. Surgical removal of the infected valve is the most effective management strategy, although not always possible. In 2008 Pai and coworkers investigated the effects of combinations of flucytosine, micafungin and voriconazole against Candida-infected human platelet-fibrin clots in an in vitro endocarditis pharmacodynamic model. Single clinical bloodstream isolates of $C$. albicans, $C$. glabrata, C. parapsilosis and $C$. tropicalis were used. Voriconazole was identified to be the least active agent, while flucytosine had the greatest activity as single agent. Micafungin was superior to voriconazole for all species except C. parapsilosis. The combination of flucytosine/ voriconazole was superior to either agent alone against $C$. parapsilosis. The combination of voriconazole/micafungin was inferior to the use of micafungin alone against $C$. tropicalis. The triple combination flucytosine/voriconazole/ micafungin was not better than single or dual agents against any of the Candida spp. [45]. As previous studies demonstrated the poor activity of voriconazole and the superior activity of flucytosine and micafungin against $C$. albicans biofilms, Pai continued his research with the investigation of 
the effects of flucytosine, liposomal amphotericin B and micafungin combinations against 2 C. albicans strains in the same endocarditis pharmacodynamic model. They studied the effects of the antifungals on $24 \mathrm{~h}$-old biofilms. By contrast with the previous study in 2008, they measured the antifungal effects only at one time point and used the standard definition of synergism. These data revealed that micafungin was uniquely active against mature C. albicans biofilms, while flucytosine was not [46]. This is in contrast to the data in the previous report, where flucytosine was markedly active against $C$. albicans biofilms [45]. The triple combination of flucytosine/liposomal amphotericin $\mathrm{B} /$ micafungin was superior to all other treatments for one isolate but not different from the dual combination liposomal amphotericin $\mathrm{B}$ /micafungin for the other isolate. Although no clear pattern of interaction between these agents was seen, the superior activity of micafungin against $24 \mathrm{~h}$-old C. albicans biofilms relative to flucytosine and liposomal amphotericin B was apparent for both isolates [46]. In conclusion, and supported by earlier reports [19], these data demonstrated the activity of echinocandins when used alone or in combination with polyenes against $C$. albicans biofilm.

In 2008, Uppuluri and coworkers demonstrated a synergistic effect of calcineurin inhibitors FK506 and cyclosporin A in combination with fluconazole against $C$. albicans biofilms in both in vitro experiments and an in vivo rat catheter model. These studies reveal that the drug synergism was mediated via direct inhibition of C. albicans calcineurin, which is known to contribute to fluconazole resistance in biofilms. [50].

Very recently, studies involving the combination of antibacterial agents with standard concentrations of antifungal agents against $C$. albicans biofilms were reported [51, 52]. In 2009, Miceli and coworkers determined the antifungal effect on C. albicans biofilms of doxycycline alone and in combination with standard combinations of amphotericin B, caspofungin and fluconazole. In this study, 128 $\mu \mathrm{g} / \mathrm{ml}$ doxycycline combined with fluconazole and 512 $\mu \mathrm{g} / \mathrm{ml}$ doxycycline combined with low doses of amphotericin B $(\leq 0.25 \mu \mathrm{g} / \mathrm{ml})$ demonstrated synergism. The paradoxical effect of high concentrations of caspofungin (16 $\mu \mathrm{g} / \mathrm{ml}$ ) was significantly attenuated in combination with 2048 and $512 \mu \mathrm{g} / \mathrm{ml}$ doxycycline. Other combinations and concentrations did not show significant positive effects against the biofilms [51]. Because of the interesting results of combination of antifungals with doxycycline, $\mathrm{Ku}$ and coworkers determined in 2010 the activity of other antibacterial agents, including azithromycin, tigecycline and vancomycin against $C$. albicans biofilms. Tigecycline was the most active agent against mature $C$. albicans biofilms and substantially inhibited the formation of the biofilms. Therefore they focused on the combination of tigecycline with amphotericin B, caspofungin and fluconazole. However, addition of tigecycline did not potentiate the effects of the antifungals tested and in some cases, inhibited their effects [52]. In another study, Shi and coworkers demonstrated the synergism between fluconazole and minocycline against fluconazole-resistant $C$. albicans isolates. The mechanism of action was suggested to be the enhancement of minocycline on fluconazole penetrating through the biofilm as well as interrupting the calcium balance instead of impacting on the uptake and efflux on fluconazole [53].
However, it should be mentioned that there are risks of inducing bacterial resistance by using the combination of high doses antibacterial agents with standard concentrations of antifungal agents against $C$. albicans biofilms.

Synergism and antagonism are in vitro concepts that are difficult to translate into clinical practice. Clinical studies are needed but hard to perform. Nevertheless, evidence of synergism of antifungal combination therapy in vitro might be the first step in establishing appropriate antifungal therapy.

\section{Antifungal Lock Therapy}

In case of implant-related infections, the guidelines for the treatment of candidiasis strongly recommend the removal or replacement of catheters and medical devices whenever feasible [14]. However, in many patients with biomaterial- or catheter-related Candida infection, removal and/or replacement of the infected device is difficult or a high risk. As an alternative strategy, antimicrobial lock therapy (ALT) has been proposed for the prevention and treatment of catheterrelated bloodstream infections, often associated with biofilm formation [19]. The guidelines for the treatment of candidiasis also state that the role for antifungal lock solutions is not well defined. The ALT involves introducing 2 to $4 \mathrm{ml}$ of a highly concentrated antimicrobial solution (100-1000x MIC or its usual target systemic concentration) into a catheter lumen and allowing the solution to dwell (lock) for a specific period of time while the catheter is not in use, in order to affect the biofilm and sterilize the lumen [54,55]. Microorganisms most frequently associated with catheter-related bloodstream infections are Staphylococcus epidermis, S. aureus and Candida species [56,57], and these microbes have been isolated together in biofilm-related polymicrobial infections [58]. The reader is directed to the review by Rizk et al. in this special issue of Open Mycology for more information on polymicrobial biofilms. A variety of antibiotics have been used as prophylactic antimicrobial catheter lock solution, including gentamycin, cefazolin/ gentamycin, minocycline or cefotaxime, and were effective in preventing catheter related bacteraemia [59-63]. In the reviewed studies by Korbila and coworkers, there were no serious adverse effects, such as emergence of resistance or increased infectious complications found to be associated with the use of ALT [64]. However, Allon and coworkers are concerned about the use of antibiotics because of the potential development of resistance and the risk for systemic toxicity due to solution leak from the catheter lumen into the circulation [65]. Non-toxic anti-biofilm agents are therefore urgently needed.

In 2003, Raad and coworkers, investigated the in vitro activities of different flush solutions against $S$. epidermis, $S$. aureus and C. albicans biofilms grown on catheter surfaces. They used a Modified Robbins device [28] as in vitro model whereby the catheter segments were flushed for $18 \mathrm{~h}$ with streptokinase, heparin, vancomycin, vancomycin/heparin, EDTA, minocycline or minocycline/EDTA [66]. EDTA is a chelator of calcium and magnesium with established anticoagulant activity and inhibitory activity against Staphyloccoci and Candida spp., which shows better results than heparin when used in flush solutions [66-70]. Minocycline is a broad spectrum tetracycline antibiotic. Only 
EDTA resulted in partial reduction of catheter colonization. As previously reported, this study shows also the failure of vancomycin and heparin against catheter-related biofilms. The combination of EDTA with low minocycline concentration $(0.1 \mathrm{mg} / \mathrm{ml})$ resulted in a significant decrease in catheter colonization but combined with higher concentrations of minocycline resulted in complete eradication of $C$. albicans biofilms. These data support other reports involving the combination of minocycline and EDTA as effective lock solution against central-venous-catheter-associated biofilms of clinically relevant microorganisms in clinical trials [71,72] and a rabbit model [73]. Another study shows that tetrasodium EDTA applied for $21 \mathrm{~h}$ to $25 \mathrm{~h}$ reduced the biofilm formation of different strains, including $C$. albicans [74]. Based on in vitro, animal and clinical studies, for an ALT to be effective, a dwell time of at least $4 \mathrm{~h}$ daily is often required $[55,68,71,75,76]$. This might not be feasible among patients who continuously require fluids and therapeutic agents through the catheters. As ethanol was shown to have only a dwell time for $1 \mathrm{~h} \mathrm{[77],} \mathrm{more} \mathrm{studies} \mathrm{involving} \mathrm{the} \mathrm{use}$ of ethanol alone or in combination with other agents were reported. Maki and coworkers showed in addition that ethanol does not alter the mechanical properties of silicone and polyurethane catheters [77]. Although low concentrations of ethanol (1-6\%) stimulate biofilm formation in some strains of $S$. epidermis [78], $10 \%$ ethanol significantly inhibited Candida biofilm formation in vitro with complete inhibition at concentrations $\geq 20 \%$. In 2009, Balestrino and coworkers reported data involving a lock solution based on $60 \%$ ethanol. This in vitro study demonstrated the superior antimicrobial activity of $60 \%$ ethanol in contrast to $47.6 \%$ trisodium citrate in eradicating monomicrobial biofilms including $C$. albicans biofilms formed on silicone catheters. A $20 \mathrm{~min} 60 \%$ ethanol-treatment completely eradicated the 4- and 24h-old C. albicans biofilms [79]. These results are in line with an earlier report [80]. The advantage of using ethanol as a lock solution in addition to the antimicrobial activity, is the low cost and universal availability. There is no evidence of acquired resistance to concentrated ethanol despite the extensive and longstanding use as an antiseptic, nor are there any studies showing hypersensitivity related to ethanol administration. Preliminary reports suggest that ethanol as a lock solution can be used without severe side effects [81-84].

In 2007, Raad and coworkers performed a study involving different combinations of minocycline, EDTA and $25 \%$ ethanol. In this study, the triple combination of minocycline/ EDTA and a low concentration of ethanol (25\%) was the only lock solution of the study that completely eradicated and completely prevented the regrowth of the biofilms of 2 Candida strains in various biofilm models. In addition this lock solution was more effective in rapidly eradicating the growth or regrowth as compared to other combinations of the agents [85].

Venkatesh and coworkers investigated the effects of different agents including ethanol on monomicrobial and polymicrobial biofilms of $C$. albicans and $S$. epidermis formed in a microtiter plate model. The minimal biofilm eradicating concentration causing 50\% inhibition (MBEC50) for ethanol was $12.5 \%$. This was the only agent that reduced the viability of $C$. albicans in polymicrobial biofilms [86]. These results are in line with previously reported data involving the effectiveness of higher concentrations of ethanol (25-70\%) against biofilms [80,85].

The in vitro anti-biofilm activity of echinocandins has been previously demonstrated, however the potential of echinocandins used as a lock therapy is poorly documented. In 2008, Cateau and coworkers investigated the in vitro efficacies of caspofungin or micafungin catheter lock solutions on $C$. albicans biofilm growth. The results demonstrated that caspofungin or micafungin used as a lock solution significantly reduced at least by half the metabolic activity of intermediate $(12 \mathrm{~h})$ and mature (5 days) biofilms of $C$. albicans and that the reduction was maintained after $48 \mathrm{~h}$ [87]. So echinocandins may have a real anti-biofilm potential and could become important factors in the lock approach.

There are different reports involving liposomal amphotericin $\mathrm{B}$ as antifungal lock therapy against $C$. albicans biofilms in a catheter infection model. The first clinical relevant model of $C$. albicans biofilms-associated catheter infection was described by Schinabeck and coworkers in 2004. They evaluated the effectiveness of liposomal amphotericin B (3 mg per day) antifungal lock therapy compared to a fluconazole ( $3 \mathrm{mg}$ per day) lock and untreated controls against $C$. albicans biofilms (3 days old) formed on silicone catheters in rabbits. The antifungal solutions were locked in the lumen of each catheter for $8 \mathrm{~h}$ per day for 7 days after 3 days post infection. Results showed that catheters treated with liposomal amphotericin B were completely clear except for 1 or 2 small patches of dead $C$. albicans surrounded by minimal amounts of damaged matrix [88]. These results are in line with earlier reports with successful salvage of catheters infected with $C$. albicans by using amphotericin $\mathrm{B}$ deoxycholate antifungal lock therapy $[89,90]$. As in the case of these reports, the treatment period of amphotericin B may be extended to 10 to 14 days for complete eradication of fungal elements of the catheters. The fluconazole treatment resulted in less biofilm compared to the untreated controls, but the biofilm structure appeared similar to that of the controls, unlike the amphotericin B treatment. Increased concentrations of fluconazole may improve its efficacy as an antifungal lock solution. However, dissolving higher concentrations in this study was difficult. Even though fluconazole lock therapy is unable to sterilize the intraluminal surface of catheters, only 1 out of 7 catheter drawn blood cultures grew yeast after the treatment period. The fluconazole solution may only kill free-floating planktonic $C$. albicans cells released from the mature biofilm. Therefore fluconazole lock therapy may represent an effective suppressive therapy for preventing continuous seeding of the bloodstream, although the infection may return when the therapy is stopped [88].

A few years later, Shuford and coworkers evaluated caspofungin and amphotericin $\mathrm{B}$ deoxycholate against $C$. albicans biofilms in a rabbit catheter infection model [25]. Their study demonstrated the effectiveness of amphotericin $\mathrm{B}$ and more specifically caspofungin, in the combination of systemic and intraluminal lock therapy against $C$. albicans biofilms associated with intraluminal and extraluminal surfaces of catheters. However, the continuous lock with amphotericin B over the treatment duration of 7 days is not practical in a clinical setting, where catheters are placed to facilitate frequent delivery of medications and/or nutrition 
and which is not possible if the catheter is continuously locked. In 2009, these data were confirmed in an independent study by Mukherjee and coworkers [26].

In conclusion, all these data demonstrate that liposomal amphotericin B lock therapy is an effective approach for treating $C$. albicans catheter-associated biofilms in a short treatment period. These findings may have significant clinical implications and warrant clinical evaluation.

\section{Novel Anti-Biofilm Agents}

The development of new anti-biofilm agents is urgently needed as the number of therapeutic options for Candida biofilm-related diseases is very small. There is a novel trend in the anti-biofilm research area towards the identification of natural products, plants and their extracts with anti-biofilm activity. In this respect, xanthorrhizol isolated from Curcuma xanthorrhiza [91] and the oil of Boesenbergia pandurata rhizomes [92] and Ocimum americanum [93] showed potent in vitro activity against Candida biofilms. In 2008, 30 plant oils were tested for their activity against $C$. albicans biofilms [94]. Peppermint, eucalyptus, ginger grass and clove oils resulted in a reduction in C. albicans biofilm formation. The main component of eucalyptus oil, 1,8-cineole, showed potent anti-biofilm activity against $C$. albicans biofilms [95]. The antifungal activity of tea tree oil has been recently studied by De Prijck and coworkers against $C$. albicans biofilms. The tea tree oil was released from modified polydimethyl siloxane disk as a model for incorporating antifungals in medical devices to prevent biofilm formation by Candida spp. The biofilm inhibition amounted to more than one log unit in the Modified Robbins Device [28] on disks impregnated with tea tree oil [96]. Also the strong antifungal activity of terpenes, which are major components of essential oils, has been described [97-99]. In 2008, 10 terpenic derivatives, corresponding to major components of essential oils, were tested for their activity against $C$. albicans biofilms. Almost all the studied terpenic derivatives showed anti-biofilm activity. However, carvacrol, geraniol and thymol exhibited the strongest activity. Moreover, these compounds also proved to be efficient against biofilms of $C$. glabrata and C. parapsilosis [100].

Chitosan recently proved to be active against in vitro Candida biofilms as well. Chitosan is a hydrophilic biopolymer that is industrially obtained by means of $N$-deacetylation of crustacean chitin. It was already known that chitosan exhibits antimicrobial activity against fungi, bacteria and viruses [101]. Now, it was determined that mature $C$. albicans and $C$. parapsilosis biofilms are susceptible to chitosan in vitro. Chitosan decreased the metabolic activity and survival of Candida species biofilms, with more than $95 \%$ killing of the sessile cells after $0.5 \mathrm{~h}$ treatment with 2.5 $\mathrm{mg} / \mathrm{ml}$ chitosan [102]. Also various saponins [103] and polyphenols [104] were shown to affect $C$. albicans biofilms. Saponins are secondary metabolites ubiquitously found in various plant species and specifically known for their hemolytic activity [103]. Polyphenols, extracted from green tea, also showed effects against C. albicans biofilms. Epigallocatechin-3-gallate, the most abundant polyphenol in a green tea extract, reduced the $C$. albicans biofilm metabolic activity with $80 \%$. Further investigations indicated that impairment of the yeast proteasomal activity might be involved in the mode of action of tea polyphenols against $C$. albicans [104]. Bisbibenzyl compounds are exclusively found in liverworts and are known to exhibit antifungal activity [105-107]. In 2009 it was demonstrated that riccardin D, a macrocyclic bisbibenzyl isolated from the Chinese liverwort Dumortiera hirsuta, causes a remarkable reduction in the metabolic activity of $C$. albicans biofilm cells at concentrations that are not toxic for RPE1 or LO2 cell lines $[108,109]$. Furthermore, it was shown that riccardin $\mathrm{D}$ acts by down regulating the expression of Candida hyphae specific genes [109]. In addition, (R)-goniothalamin, the most abundant styryl lactone in the Goniothalamus genus (Annonaceae family), is active against $C$. albicans biofilms [110]. It was already reported that $(R)$-goniothalamin as well as its non-natural enantiomer $(S)$-goniothalamin, exhibit antifungal activity against human pathogenic fungi and have minimal toxic effects on mammalian cell lines [111].

Carbazoles are aromatic heterocyclic organic compounds first isolated from coal tar [112]. Carbazole alkaloids have been isolated form various plants and microorganisms. In a recently conducted compound screening, aimed at finding novel fungicidal compounds, a series of substituted carbazoles (N-alkylated 3,6-dihalogenocarbazoles) was identified. Besides their fungicidal activity against planktonic $C$. albicans and C. glabrata cells, some of them were also active against Candida biofilms grown in microtiter plates [113].

Besides echinocandins, various other naturally occurring peptides have been found with potent activity against Candida biofilms, including lactoferrin and histatins. Lactoferrin is an iron-binding glycoprotein, which is naturally present in human glandular secretions (milk, tears and salvia), and has a broad-spectrum antimicrobial activity against bacteria and fungi [114]. Venkatesh and coworkers observed that talactoferrin, human recombinant lactoferrin, significantly decreased the biomass and thickness of $S$. epidermis and C. albicans biofilms [86].

Histatins, a family of histidine-rich cationic peptides secreted by the major salivary glands in humans, especially histatin 5, possess significant antifungal properties [115]. A recent study, demonstrated that histatin 5 exhibits antifungal activity against biofilms of $C$. albicans and to a lesser extent against biofilms of $C$. glabrata developed on denture acrylic [116]. Naturally occurring antimicrobial peptides hold promise as therapeutic agents against pathogens such as $C$. albicans but numerous difficulties have slowed their development. They are difficult and expensive to produce in large quantities and are often sensitive to protease digestion [117]. The quest for new and improved antimicrobial peptides has led to the study of peptide mimetics. Synthetic analogs that mimic the properties of these peptides have many advantages and exhibit potent, selective antimicrobial activity [118]. In 2002, Stark and coworkers developed a new class of antimicrobial peptides that were originally designed to mimic transmembrane segments of integral membrane proteins and were tagged with lysine residues to facilitate solubilization in aqueous media. The peptides, designated kaxins, have a non-amphipathic hydrophobic core segment, which distinguishes them from many natural linear cationic antimicrobial peptides. They showed that placing all of the $\mathrm{K}$ residues on the $\mathrm{N}$-terminus and generating all-D- 
enantiomeric versions, in combination with decreasing the length of the hydrophobic segment, resulted in shorter (and therefore less expensive) peptides that generally displayed increased antimicrobial activity [119]. In 2006, Burrows and coworkers tested the antifungal activity of a subset of those peptides with a good antimicrobial activity without mammalian cell toxicity, and new deratives thereof. They performed different assays including the ability of 2 peptides (dF17-6K and $\mathrm{dF} 21-10 \mathrm{~K}$ ) with the best antifungal activity to kill biofilms of $C$. albicans and C. tropicalis [120]. The amount of peptide required for eradication was less than the 30-2000x MIC required for small molecule antifungals to kill biofilms [121]. The amount of peptide may be reduced even further by attaching the peptides directly to the surface of biomaterials to prevent biofilm formation at the stage of initial attachment, as shown to be successful for long-chain hydrophobic polycations, which are mimics of antimicrobial peptides $[122,123]$.

Based on the antifungal activity of $\beta$-peptides ( $\beta$-amino acid oligomers) against planktonic $C$. albicans, Karlsson and coworkers demonstrated in 2009 that these $\beta$-peptides at a concentration near the minimum inhibitory concentration completely prevented planktonic cells to form a biofilm by a toxicity mechanism involving membrane disruption [124]. Recently, the fabrication of multilayered polyelectrolyte thin films that promote the surface-mediated release of an antifungal $\beta$-peptide was reported [125]. The films inhibited the growth of $C$. albicans on film-coated surfaces, resulting in a $20 \%$ reduction of cell viability after $2 \mathrm{~h}$ and a $74 \%$ decrease in metabolic activity after $7 \mathrm{~h}$ when compared to cells incubated on coated surfaces without $\beta$-peptide. In addition, $\beta$-peptide-containing films inhibited hyphal elongation by $55 \%$. This approach could ultimately be used to coat the surfaces of catheters, surgical instruments, and other devices to inhibit drug-resistant $C$. albicans biofilm formation in clinical settings [125]. In addition, Hua and coworkers screened several series of antimicrobial peptide mimetics (with molecular weight $<1000 \mathrm{Da}$ ) against oral Candida strains. One phenylalkyne and several arylamide compounds with reduced mammalian cytotoxicities were found to be active against $C$. albicans. These compounds demonstrated rapid fungicidal activity in liquid culture even in the presence of saliva, and demonstrated synergy with standard antifungal agents. When assayed against biofilms grown on denture acrylic, the compounds exhibited potent fungicidal activity. Repeated passages in sub-minimum inhibitory concentration levels did not lead to resistant Candida, in contrast to fluconazole. These results demonstrate the proof-of principle for the use of these compounds as anti-Candida agents, and their further testing is warranted as novel anti-Candida therapies [126].

\section{CONCLUSIONS}

Candida biofilms are intrinsically resistance to most antifungal drugs. However, the lipid formulations of amphotericin $\mathrm{B}$ and the echinocandins, used alone or in combination with the polyenes, do have activity against Candida biofilms. As an alternative strategy, antimicrobial lock therapy (ALT) has been proposed for the prevention and treatment of catheter-related bloodstream infections, often associated with biofilm formation. In this respect, liposomal amphotericin B lock therapy is an effective approach for treating $C$. albicans catheter-associated biofilms in a short treatment period. However, the development of new antibiofilm agents is urgently needed since the number of therapeutic options for Candida biofilm-related diseases is very small. Furthermore, there is an increasing awareness of the hazards that are associated with the use of antibiotic and chemical agents. This has led to accelerated investigations on natural products, plants and their extracts as new sources of antimicrobial agents. The exploration of new and effective natural compounds with antifungal activity against $C$. albicans biofilm cells and with low cytotoxicity is likely to have a significant impact on the treatment and management of biofilm-associated fungal infections.

\section{REFERENCES}

[1] Nucci M, Anaissie E. Revisiting the source of candidemia: skin or gut? Clin Infect Dis 2001; 33(12): 1959-67.

[2] Douglas JL, Cobbs CG. Prosthetic valve endocarditis. In: Kaye D, Ed. Infective endocarditis. 2d ed. New York: Raven Press 1992; 375-96.

[3] Tunney MM, Patrick S, Curran MD, et al. Detection of prosthetic joint biofilm infection using immunological and molecular techniques. Methods Enzymol 1999; 310: 566-76.

[4] Raad I, Costerton W, Sabharwal U, Sacilowski M, Anaissie E, Bodey GP. Ultrastructural analysis of indwelling vascular catheters: a quantitative relationship between luminal colonization and duration of placement. J Infect Dis 1993; 168(2): 400-7.

[5] Ramage G, Martínez JP, López-Ribot JL. Candida biofilms on implanted biomaterials: a clinically significant problem. FEMS Yeast Res 2006; 6(7): 979-86.

[6] Seneviratne CJ, Jin L, Samaranayake LP. Biofilm lifestyle of Candida: a mini review. Oral Dis 2008; 14(7): 582-90.

[7] LaFleur M, Kumamoto CA and Lewis K. Candida albicans biofilms produce antifungal-tolerant persister cells. Antimicrob Agents Chemother 2006; 50: 3839-46.

[8] Lewis, K. Persister cells. Annu Rev Microbiol 2010; 64: 357-72.

[9] Hawser SP, Douglas LJ. Resistance of Candida albicans biofilms to antifungal agents in vitro. Antimicrob Agents Chemother 1995; 39(9): 2128-31.

[10] Mermel LA, Farr BM, Sherertz RJ, et al. Infectious Diseases Society of America; American College of Critical Care Medicine; Society for Healthcare Epidemiology of America. Guidelines for the management of intravascular catheter-related infections. Clin Infect Dis 2001; 32(9): 1249-72.

[11] Mermel LA, Allon M, Bouza E, et al. Clinical practice guidelines for the diagnosis and management of intravascular catheter-related infection. Clin Infect Dis 2009; 49(1): 1-45.

[12] Nguyen MH, Peacock JE, Tanner DC, Morris AJ, Nguyen ML, Snydman DR, Wagener MM, Yu VL. Therapeutic approaches in patients with candidemia: evaluation in a multicenter prospective observational study. Arch Intern Med 1995; 155: 2429-35.

[13] Nucci M, Colombo AL, Silveira F, et al. Risk factors for death in patients with candidemia. Infect Control Hosp Epidemiol 1998; 19: 846-50.

[14] Pappas PG, Kauffman CA, Andes D, et al. Infectious Diseases Society of America. Clinical practice guidelines for the management of candidiasis. Clin Infect Dis 2009; 48(5): 503-35.

[15] Rex JH, Pfaller MA, Galgiani JN, Bartlett MS, Espinel-Ingroff A, Ghannoum MA, Lancaster M, Odds FC, Rinaldi MG, Walsh TJ, Barry AL. Development of interpretive breakpoints for antifungal susceptibility testing: conceptual framework and analysis of in vitro-in vivo correlation data for fluconazole, itraconazole, and candida infections. Clin Infect Dis 1997; 24(2): 235-47.

[16] Baillie GS, Douglas LJ. Candida biofilms and their susceptibility to antifungal agents. Methods Enzymol 1999; 310: 644-56.

[17] Chandra J, Mukherjee PK, Leidich SD, Faddoul FF, Hoyer LL, Douglas LJ, Ghannoum MA. Antifungal resistance of candidal biofilms formed on denture acrylic in vitro. J Dent Res 2001; 80(3): 903-8. 
[18] Hawser SP, Douglas LJ. Biofilm formation by Candida species on the surface of catheter materials in vitro. Infect Immun 1994; 62(3): 915-21.

[19] Kuhn DM, George T, Chandra J, Mukherjee PK, Ghannoum MA. Antifungal susceptibility of Candida biofilms: unique efficacy of amphotericin B lipid formulations and echinocandins. Antimicrob Agents Chemother 2002; 46(6): 1773-80.

[20] Lamfon H, Porter SR, McCullough M, Pratten J. Susceptibility of Candida albicans biofilms grown in a constant depth film fermentor to chlorhexidine, fluconazole and miconazole: a longitudinal study. J Antimicrob Chemother 2004; 53(2): 383-5.

[21] Vandenbosch D, Braeckmans K, Nelis HJ, Coenye T. Fungicidal activity of miconazole against Candida spp. biofilms. J Antimicrob Chemother 2010; 65(4): 694-700.

[22] Katragkou A, Chatzimoschou A, Simitsopoulou M, et al. Differential activities of newer antifungal agents against Candida albicans and Candida parapsilosis biofilms. Antimicrob Agents Chemother 2008; 52(1): 357-60.

[23] Hawser SP, Norris H, Jessup CJ, Ghannoum MA. Comparison of a 2,3-bis(2-methoxy-4-nitro-5-sulfophenyl)-5-

[(phenylamino)carbonyl]-2H-tetrazolium hydroxide (XTT) colorimetric method with the standardized National Committee for Clinical Laboratory Standards method of testing clinical yeast isolates for susceptibility to antifungal agents. J Clin Microbiol 1998; 36(5): 1450-2.

[24] Seidler M, Salvenmoser S, Müller FM. Liposomal amphotericin B eradicates Candida albicans biofilm in a continuous catheter flow model. FEMS Yeast Res 2010; 10(4): 492-5.

[25] Shuford JA, Rouse MS, Piper KE, Steckelberg JM, Patel R. Evaluation of caspofungin and amphotericin B deoxycholate against Candida albicans biofilms in an experimental intravascular catheter infection model. J Infect Dis 2006; 194(5): 710-3.

[26] Mukherjee PK, Long L, Kim HG, Ghannoum MA. Amphotericin B lipid complex is efficacious in the treatment of Candida albicans biofilms using a model of catheter-associated Candida biofilms. Int J Antimicrob Agents 2009; 33(2): 149-53.

[27] Redding S, Bhatt B, Rawls HR, Siegel G, Scott K, Lopez-Ribot J. Inhibition of Candida albicans biofilm formation on denture material. Oral Surg Oral Med Oral Pathol Oral Radiol Endod 2009; 107(5): 669-72.

[28] Raad I, Darouiche R, Hachem R, Sacilowski M, Bodey GP. Antibiotics and prevention of microbial colonization of catheters. Antimicrob Agents Chemother 1995; 39(11): 2397-400.

[29] De Prijck K, De Smet N, Honraet K, et al. Inhibition of Candida albicans biofilm formation by antimycotics released from modified polydimethyl siloxane. Mycopathologia 2010; 169(3): 167-74.

[30] Ramage G, Vande Walle K, Wickes BL, López-Ribot JL. Standardized method for in vitro antifungal susceptibility testing of Candida albicans biofilms. Antimicrob Agents Chemother 2001; 45(9): 2475-9.

[31] Bachmann SP, VandeWalle K, Ramage G, et al. In vitro activity of caspofungin against Candida albicans biofilms. Antimicrob Agents Chemother 2002; 46(11): 3591-6.

[32] Stone JA, Holland SD, Wickersham PJ, et al. Single- and multipledose pharmacokinetics of caspofungin in healthy men. Antimicrob Agents Chemother 2002; 46(3): 739-45.

[33] Cocuaud C, Rodier MH, Daniault G, Imbert C. Anti-metabolic activity of caspofungin against Candida albicans and Candida parapsilosis biofilms. J Antimicrob Chemother 2005; 56(3): 50712.

[34] Seidler M, Salvenmoser S, Müller FM. In vitro effects of micafungin against Candida biofilms on polystyrene and central venous catheter sections. Int J Antimicrob Agents 2006; 28(6): 568-73.

[35] Choi HW, Shin JH, Jung SI, et al. Species-specific differences in the susceptibilities of biofilms formed by Candida bloodstream isolates to echinocandin antifungals. Antimicrob Agents Chemother 2007; 51(4): 1520-3.

[36] Lazzell AL, Chaturvedi AK, Pierce CG, Prasad D, Uppuluri P, Lopez-Ribot JL. Treatment and prevention of Candida albicans biofilms with caspofungin in a novel central venous catheter murine model of candidiasis. J Antimicrob Chemother 2009; 64(3): 567-70.

[37] Ricicová M, Kucharíková S, Tournu H, et al. Candida albicans biofilm formation in a new in vivo rat model. Microbiology 2010; 156: 909-19.
[38] Kucharíková S, Tournu H, Holtappels M, Van Dijck P, Lagrou K. In vivo efficacy of anidulafungin against Candida albicans mature biofilms in a novel rat model of catheter-associated candidiasis. Antimicrob Agents Chemother 2010; 54(10): 4474-5.

[39] Lewis RE, Kontoyiannis DP. Rationale for combination antifungal therapy. Pharmacotherapy 2001; 21: 149S-164S.

[40] Eliopoulos GM, Moellering RC. In: V Lorian, Antibiotics in laboratory medicine, $3^{\text {rd }} \mathrm{cd}$. Antimicrobial combinations. Baltimore, The Williams \& Wilkins Co. 1991; 432-92.

[41] Mukherjee PK, Sheehan DJ, Hitchcock CA, Ghannoum MA. Combination treatment of invasive fungal infections. Clin Microbiol Rev 2005; 18(1): 163-94.

[42] Bachmann SP, Ramage G, VandeWalle K, Patterson TF, Wickes BL, López-Ribot JL. Antifungal combinations against Candida albicans biofilms in vitro. Antimicrob Agents Chemother 2003; 47(11): 3657-9.

[43] Shuford JA, Piper KE, Steckelberg JM, Patel R. In vitro biofilm characterization and activity of antifungal agents alone and in combination against sessile and planktonic clinical Candida albicans isolates. Diagn Microbiol Infect Dis 2007; 57(3): 277-81.

[44] Tobudic S, Lassnigg A, Kratzer C, Graninger W, Presterl E. Antifungal activity of amphotericin B, caspofungin and posaconazole on Candida albicans biofilms in intermediate and mature development phases. Mycoses 2010; 53(3): 208-14.

[45] Pai MP, Samples ML, Mercier RC, Spilde MN. Activities and ultrastructural effects of antifungal combinations against simulated Candida endocardial vegetations. Antimicrob Agents Chemother 2008; 52(7): 2367-76.

[46] Pai MP. Antifungal combinations against simulated Candida albicans endocardial vegetations. Antimicrob Agents Chemother 2009; 53(6): 2629-31.

[47] Baddour LM, Wilson WR, Bayer AS, et al. Infective endocarditis: diagnosis, antimicrobial therapy, and management of complications: a statement for healthcare professionals from the Committee on Rheumatic Fever, Endocarditis, and Kawasaki Disease, Council on Cardiovascular Disease in the Young, and the Councils on Clinical Cardiology, Stroke, and Cardiovascular Surgery and Anesthesia, American Heart Association: endorsed by the Infectious Diseases Society of America. 2005;111(23): e394434.

[48] Pappas PG. Invasive candidiasis. Infect Dis Clin North Am 2006; 20(3): 485-506.

[49] Moudgal V, Little T, Boikov D, Vazquez JA. Multiechinocandinand multiazole-resistant Candida parapsilosis isolates serially obtained during therapy for prosthetic valve endocarditis. Antimicrob Agents Chemother 2005; 49(2): 767-9.

[50] Uppuluri P, Nett J, Heitman J, Andes D. Synergistic effect of calcineurin inhibitors and fluconazole against Candida albicans biofilms. Antimicrob Agents Chemother 2008; 52(3): 1127-32.

[51] Miceli MH, Bernardo SM, Lee SA. In vitro analyses of the combination of high-dose doxycycline and antifungal agents against Candida albicans biofilms. Int J Antimicrob Agents 2009; 34(4): 326-32.

[52] Ku TS, Palanisamy SK, Lee SA. Susceptibility of Candida albicans biofilms to azithromycin, tigecycline and vancomycin and the interaction between tigecycline and antifungals. Int $\mathrm{J}$ Antimicrob Agents 2010; 36(5): 441-6.

[53] Shi W, Chen Z, Chen X, Cao L, Liu P, Sun S. The combination of minocycline and fluconazole causes synergistic growth inhibition against Candida albicans: an in vitro interaction of antifungal and antibacterial agents. FEMS Yeast Res 2010; 10(7): 885-93.

[54] Carratalà J. The antibiotic-lock technique for therapy of 'highly needed' infected catheters. Clin Microbiol Infect. 2002; 8(5): 282-9. Shah CB, Mittelman MW, Costerton JW, et al. Antimicrobial activity of a novel catheter lock solution. Antimicrob Agents Chemother 2002; 46(6): 1674-9.

[56] Raad II, Hanna HA. Intravascular catheter-related infections: new horizons and recent advances. Arch Intern Med 2002; 162(8): 8718.

[57] Sherertz RJ, Raad II, Belani A, et al. Three-year experience with sonicated vascular catheter cultures in a clinical microbiology laboratory. J Clin Microbiol 1990; 28(1): 76-82.

[58] Karlowicz MG, Hashimoto LN, Kelly RE Jr, Buescher ES. Should central venous catheters be removed as soon as candidemia is detected in neonates? Pediatrics 2000; 106(5): E63. 
[59] Dogra GK, Herson H, Hutchison B, et al. Prevention of tunneled hemodialysis catheter-related infections using catheter-restricted filling with gentamicin and citrate: a randomized controlled study. J Am Soc Nephrol 2002; 13(8): 2133-9.

[60] Jaffer Y, Selby NM, Taal MW, Fluck RJ, McIntyre CW. A metaanalysis of hemodialysis catheter locking solutions in the prevention of catheter-related infection. Am J Kidney Dis 2008; 51(2): 233-41

[61] McIntyre CW, Hulme LJ, Taal M, Fluck RJ. Locking of tunneled hemodialysis catheters with gentamicin and heparin. Kidney Int 2004; 66(2): 801-5.

[62] Nori US, Manoharan A, Yee J, Besarab A. Comparison of lowdose gentamicin with minocycline as catheter lock solutions in the prevention of catheter-related bacteremia. Am J Kidney Dis 2006; 48(4): 596-605.

[63] Saxena AK, Panhotra BR, Sundaram DS, Morsy MN, Al-Ghamdi AM. Enhancing the survival of tunneled haemodialysis catheters using an antibiotic lock in the elderly: a randomised, double-blind clinical trial. Nephrology 2006; 11(4): 299-305.

[64] Korbila IP, Bliziotis IA, Lawrence KR, Falagas ME. Antibioticlock therapy for long-term catheter-related bacteremia: a review of the current evidence. Expert Rev Anti Infect Ther 2007; 5(4): 63952.

[65] Allon M. Prophylaxis against dialysis catheter-related bacteremia: a glimmer of hope. Am J Kidney Dis 2008; 51(2): 165-8.

[66] Raad I, Chatzinikolaou I, Chaiban G, et al. In vitro and ex vivo activities of minocycline and EDTA against microorganisms embedded in biofilm on catheter surfaces. Antimicrob Agents Chemother 2003; 47(11): 3580-5.

[67] Chatzinikolaou I, Zipf TF, Hanna H, et al. Minocyclineethylenediaminetetraacetate lock solution for the prevention of implantable port infections in children with cancer. Clin Infect Dis 2003; 36(1): 116-9.

[68] Gil ML, Casanova M, Martínez JP. Changes in the cell wall glycoprotein composition of Candida albicans associated to the inhibition of germ tube formation by EDTA. Arch Microbiol 1994; 161(6): 489-94.

[69] Reardon DM, Warner B, Trowbridge EA. EDTA, the traditional anticoagulant of haematology: with increased automation is it time for a review? Med Lab Sci 1991; 48(1): 72-5.

[70] Root JL, McIntyre OR, Jacobs NJ, Daghlian CP. Inhibitory effect of disodium EDTA upon the growth of Staphylococcus epidermidis in vitro: relation to infection prophylaxis of Hickman catheters. Antimicrob Agents Chemother 1988; 32(11): 1627-31.

[71] Evans RC, Holmes CJ. Effect of vancomycin hydrochloride on Staphylococcus epidermidis biofilm associated with silicone elastomer. Antimicrob Agents Chemother 1987; 31(6): 889-94.

[72] Bleyer AJ, Mason L, Russell G, Raad II, Sherertz RJ. A randomized, controlled trial of a new vascular catheter flush solution (minocycline-EDTA) in temporary hemodialysis access. Infect Control Hosp Epidemiol 2005; 26(6): 520-4.

[73] Raad I, Hachem R, Tcholakian RK, Sherertz R. Efficacy of minocycline and EDTA lock solution in preventing catheter-related bacteremia, septic phlebitis, and endocarditis in rabbits. Antimicrob Agents Chemother 2002; 46(2): 327-32.

[74] Percival SL, Kite P, Eastwood K, et al. Tetrasodium EDTA as a novel central venous catheter lock solution against biofilm. Infect Control Hosp Epidemiol 2005; 26(6): 515-9.

[75] Koldehoff M, Zakrzewski JL. Taurolidine is effective in the treatment of central venous catheter-related bloodstream infections in cancer patients. Int J Antimicrob Agents 2004; 24(5): 491-5.

[76] Poole CV, Carlton D, Bimbo L, Allon M. Treatment of catheterrelated bacteraemia with an antibiotic lock protocol: effect of bacterial pathogen. Nephrol Dial Transplant 2004; 19(5): 1237-44.

[77] Crnich CJ, Halfmann JA, Crone WC, Maki DG. The effects of prolonged ethanol exposure on the mechanical properties of polyurethane and silicone catheters used for intravascular access. Infect Control Hosp Epidemiol 2005; 26(8): 708-14.

[78] Knobloch JK, Horstkotte MA, Rohde H, Kaulfers PM, Mack D. Alcoholic ingredients in skin disinfectants increase biofilm expression of Staphylococcus epidermidis. J Antimicrob Chemother 2002; 49(4): 683-7.

[79] Balestrino D, Souweine B, Charbonnel N, et al. Eradication of microorganisms embedded in biofilm by an ethanol-based catheter lock solution. Nephrol Dial Transplant 2009; 24(10): 3204-9.
[80] Chambers ST, Peddie B, Pithie A. Ethanol disinfection of plasticadherent micro-organisms. J Hosp Infect 2006; 63(2): 193-6.

[81] Ackoundou-N'guessan C, Heng AE, Guenu S, et al. Ethanol lock solution as an adjunct treatment for preventing recurrent catheterrelated sepsis--first case report in dialysis setting. Nephrol Dial Transplant 2006; 21(11): 3339-40.

[82] Dannenberg C, Bierbach U, Rothe A, Beer J, Körholz D. Ethanollock technique in the treatment of bloodstream infections in pediatric oncology patients with broviac catheter. J Pediatr Hematol Oncol 2003; 25(8): 616-21.

[83] Mouw E, Chessman K, Lesher A, Tagge E. Use of an ethanol lock to prevent catheter-related infections in children with short bowel syndrome. Pediatr Surg 2008; 43(6): 1025-9.

[84] Schwab SJ, Weiss MA, Rushton F, et al. Multicenter clinical trial results with the LifeSite hemodialysis access system. Kidney Int 2002; 62(3): 1026-33.

[85] Raad I, Hanna H, Dvorak T, Chaiban G, Hachem R. Optimal antimicrobial catheter lock solution, using different combinations of minocycline, EDTA, and 25-percent ethanol, rapidly eradicates organisms embedded in biofilm. Antimicrob Agents Chemother 2007; 51(1): 78-83.

[86] Venkatesh M, Rong L, Raad I, Versalovic J. Novel synergistic antibiofilm combinations for salvage of infected catheters. J Med Microbiol2009; 58: 936-44.

[87] Cateau E, Rodier MH, Imbert CJ. In vitro efficacies of caspofungin or micafungin catheter lock solutions on Candida albicans biofilm growth. J Antimicrob Chemother 2008; 62(1): 153-5.

[88] Schinabeck MK, Long LA, Hossain MA, et al. Rabbit model of Candida albicans biofilm infection: liposomal amphotericin B antifungal lock therapy. Antimicrob Agents Chemother 2004; 48(5): 1727-32.

[89] Johnson DC, Johnson FL, Goldman S. Preliminary results treating persistent central venous catheter infections with the antibiotic lock technique in pediatric patients. Pediatr Infect Dis J 1994; 13(10): 930-1.

[90] Viale P, Petrosillo N, Signorini L, Puoti M, Carosi G. Should lock therapy always be avoided for central venous catheter-associated fungal bloodstream infections? Clin Infect Dis 2001; 33(11): $1947-$ 8.

[91] Rukayadi Y, Han S, Yong D, Hwang JK. In vitro activity of xanthorrhizol against Candida glabrata, C. guilliermondii, and C. parapsilosis biofilms. Med Mycol 2010; In Press.

[92] Taweechaisupapong S, Singhara S, Lertsatitthanakorn P, Khunkitti W. Antimicrobial effects of Boesenbergia pandurata and Piper sarmentosum leaf extracts on planktonic cells and biofilm of oral pathogens. Pak J Pharm Sci 2010; 23(2): 224-31.

[93] Thaweboon S, Thaweboon B. In vitro antimicrobial activity of Ocimum americanum $L$. essential oil against oral microorganisms. Southeast Asian J Trop Med Public Health 2009; 40(5): 1025-33.

[94] Agarwal V, Lal P, Pruthi V. Prevention of Candida albicans biofilm by plant oils. Mycopathologia 2008; 165(1): 13-9.

[95] Hendry ER, Worthington T, Conway BR, Lambert PA. Antimicrobial efficacy of eucalyptus oil and 1,8-cineole alone and in combination with chlorhexidine digluconate against microorganisms grown in planktonic and biofilm cultures. J Antimicrob Chemother 2009; 64(6): 1219-25.

[96] De Prijck K, De Smet N, Coenye T, Schacht E, Nelis HJ Prevention of Candida albicans biofilm formation by covalently bound dimethylaminoethylmethacrylate and polyethylenimine. Mycopathologia 2010; 170(4): 213-21.

[97] Mondello F, De Bernardis F, Girolamo A, Cassone A, Salvatore G. In vivo activity of terpinen-4-ol, the main bioactive component of Melaleuca alternifolia Cheel (tea tree) oil against azole-susceptible and -resistant human pathogenic Candida species. BMC Infect Dis 2006; 6: 158 .

[98] Manohar V, Ingram C, Gray J, et al. Antifungal activities of origanum oil against Candida albicans. Mol Cell Biochem 2001; 228(1-2): 111-7.

[99] Tampieri MP, Galuppi R, Macchioni F, et al. The inhibition of Candida albicans by selected essential oils and their major components. Mycopathologia 2005; 159(3): 339-45.

[100] Dalleau S, Cateau E, Bergès T, Berjeaud JM, Imbert C. In vitro activity of terpenes against Candida biofilms. Int $\mathrm{J}$ Antimicrob Agents 2008; 31(6): 572-6. 
[101] Rabea EI, Badawy ME, Stevens CV, Smagghe G, Steurbaut W. Chitosan as antimicrobial agent: applications and mode of action. Biomacromolecules 2003; 4(6): 1457-65.

[102] Martinez LR, Mihu MR, Tar M, et al. Demonstration of antibiofilm and antifungal efficacy of chitosan against candidal biofilms, using an in vivo central venous catheter model. J Infect Dis 2010; 201(9): 1436-40.

[103] Coleman JJ, Okoli I, Tegos GP, et al. Characterization of plantderived saponin natural products against Candida albicans. ACS Chem Biol 2010; 5(3): 321-32.

[104] Evensen NA, Braun PC. The effects of tea polyphenols on Candida albicans: inhibition of biofilm formation and proteasome inactivation. Can J Microbiol 2009; 55(9): 1033-9.

[105] Xie CF, Lou HX. Chemical Constituents from the Chinese Bryophytes and Their Reversal of Fungal Resistance. Curr Org Chem 2008; 12(8): 619-28.

[106] Guo XL, Leng P, Yang Y, Yu LG, Lou HX. Plagiochin E, a botanic-derived phenolic compound, reverses fungal resistance to fluconazole relating to the efflux pump. J Appl Microbiol 2008; 104(3): 831-8.

[107] Wu XZ, Cheng AX, Sun LM, Lou HX. Effect of plagiochin E, an antifungal macrocyclic bis(bibenzyl), on cell wall chitin synthesis in Candida albicans. Acta Pharmacol Sin 2008; 29(12): 1478-85.

[108] Lu ZQ, Fan PH, Ji M, Lou HX. Terpenoids and bisbibenzyls from Chinese liverworts Conocephalum conicum and Dumortiera hirsuta. J Asian Nat Prod Res 2006; 8(1-2): 187-92.

[109] Cheng A, Sun L, Wu X, Lou H. The inhibitory effect of a macrocyclic bisbibenzyl riccardin $\mathrm{D}$ on the biofilms of Candida albicans. Biol Pharm Bull 2009; 32(8): 1417-21.

[110] Martins CV, de Resende MA, da Silva DL, et al. In vitro studies of anticandidal activity of goniothalamin enantiomers. J Appl Microbiol 2009; 107(4): 1279-86.

[111] de Fátima A, Martins CVB, de Resende MA, et al. Antifungal activity of goniothalamin enantiomers. Lett Drug Des Disc 2008; 5 : 74-8.

[112] Greabe C, Glaser C. Ueber Carbazole. Ann Chem Pharm 1872; 163: 343-60.

[113] Thevissen K, Marchand A, Chaltin P, Meert EM, Cammue BP. Antifungal carbazoles. Curr Med Chem 2009; 16(17): 2205-11.
[114] Valenti P, Antonini G. Lactoferrin: an important host defence against microbial and viral attack. Cell Mol Life Sci 2005; 62(22): 2576-87.

[115] Xu T, Levitz SM, Diamond RD, Oppenheim FG. Anticandidal activity of major human salivary histatins. Infect Immun 1991; 59(8): 2549-54.

[116] Konopka K, Dorocka-Bobkowska B, Gebremedhin S, Düzgünes N Susceptibility of Candida biofilms to histatin 5 and fluconazole. Antonie Van Leeuwenhoek 2010; 97(4): 413-7.

[117] Pazgier M, Hoover DM, Yang D, Lu W, Lubkowski J. Human beta-defensins. Cell Mol Life Sci 2006; 63: 1294-313.

[118] Tew GN, Liu D, Chen B, et al. De novo design of biomimetic antimicrobial polymers. Proc Natl Acad Sci USA 2002; 99: 5110-4.

[119] Stark M, Liu LP, Deber CM. Cationic hydrophobic peptides with antimicrobial activity. Antimicrob Agents Chemother 2002; 46(11): 3585-90

[120] Burrows LL, Stark M, Chan C, Glukhov E, Sinnadurai S, Deber CM.Activity of novel non-amphipathic cationic antimicrobial peptides against Candida species. J Antimicrob Chemother 2006; 57(5): 899-907.

[121] Douglas LJ. Candida biofilms and their role in infection. Trends Microbiol 2003; 11(1): 30-6.

[122] Milović NM, Wang J, Lewis K, Klibanov AM. Immobilized Nalkylated polyethylenimine avidly kills bacteria by rupturing cell membranes with no resistance developed. Biotechnol Bioeng 2005; 90(6): 715-22.

[123] Ernsting MJ, Bonin GC, Yang M, Labow RS, Santerre JP. Generation of cell adhesive substrates using peptide fluoralkyl surface modifiers. Biomaterials 2005; 26(33): 6536-46.

[124] Karlsson AJ, Pomerantz WC, Neilsen KJ, Gellman SH, Palecek SP. Effect of sequence and structural properties on 14-helical betapeptide activity against Candida albicans planktonic cells and biofilms. ACS Chem Biol 2009; 4(7): 567-79.

[125] Karlsson AJ, Flessner RM, Gellman SH, Lynn DM, Palecek SP. Polyelectrolyte multilayers fabricated from antifungal $\beta$-peptides: design of surfaces that exhibit antifungal activity against Candida albicans. Biomacromolecules 2010; 11(9): 2321-8.

[126] Hua J, Yamarthy R, Felsenstein S, Scott RW, Markowitz K, Diamond G. Activity of antimicrobial peptide mimetics in the oral cavity: I. Activity against biofilms of Candida albicans. Mol Oral Microbiol 2010; 25(6): 418-25.

\section{Received: November 16, 2010 \\ (C) Bink et al.; Licensee Bentham Open.}

Revised: February 28, 2011

Accepted: March 16, 2011

This is an open access article licensed under the terms of the Creative Commons Attribution Non-Commercial License (http://creativecommons.org/licenses/by$\mathrm{nc} / 3.0 /$ ), which permits unrestricted, non-commercial use, distribution and reproduction in any medium, provided the work is properly cited. 